\title{
Cost-Effectiveness of the Use of Autologous Cell Harvesting Device Compared to Standard of Care for Treatment of Severe Burns in the United States
}

\author{
Stacey Kowal (D) - Eliza Kruger • Pinar Bilir · James H. Holmes IV • \\ William Hickerson · Kevin Foster · Scott Nystrom • Jeremiah Sparks • \\ Narayan Iyer · Katie Bush · Andrew Quick
}

Received: March 13, 2019 / Published online: May 7, 2019

(C) The Author(s) 2019

\begin{abstract}
Introduction: When introducing a new intervention into burn care, it is important to consider both clinical and economic impacts, as the financial burden of burns in the USA is significant. This study utilizes a health economic modeling approach to estimate cost-effectiveness and burn center budget-impact for the use of the RECELL ${ }^{\circledR}$ Autologous Cell Harvesting Device to prepare autologous skin cell suspension (ASCS) compared to standard of care (SOC)
\end{abstract}

Enhanced Digital Features To view enhanced digital features for this article go to https://doi.org/10.6084/ m9.figshare.7993028.

Electronic supplementary material The online version of this article (https://doi.org/10.1007/s12325019-00961-2) contains supplementary material, which is available to authorized users.

S. Kowal $(\varangle) \cdot$ E. Kruger · P. Bilir

IQVIA, 3110 Fairview Park Drive, Falls Church, VA, USA

e-mail: skowal@us.imshealth.com

J. H. Holmes IV

Wake Forest University Baptist Medical Center,

Winston-Salem, NC, USA

W. Hickerson

University of Tennessee Health Science Center,

Memphis, TN, USA

K. Foster

Arizona Burn Center, Phoenix, AZ, USA split-thickness skin graft (STSG) for the treatment of severe burn injuries requiring surgical intervention for definitive closure.

Methods: A hospital-perspective model using sequential decision trees depicts the acute burn care pathway (wound assessment, debridement/ excision, temporary coverage, definitive closure) and predicts the relative differences between use of ASCS compared to SOC. Clinical inputs and ASCS impact on length of stay (LOS) were derived from clinical trials and real-world use data, American Burn Association National Burn Repository database analyses, and burn surgeon interviews. Hospital resource use and unit costs were derived from three US burn centers. A budget impact calculation leverages Monte Carlo simulation to estimate the overall impact to a burn center.

Results: ASCS treatment is cost-saving or costneutral $(<2 \%$ difference) and results in lower

\section{S. Nystrom}

Office of Assistant Secretary for Preparedness and Response (ASPR), US Department of Health and Human Services (HHS), Washington, USA

\section{N. Iyer}

Biomedical Advanced Research and Development Authority (BARDA), Office of Assistant Secretary for Preparedness and Response (ASPR), US Department of Health and Human Services (HHS), Washington, USA

J. Sparks · K. Bush · A. Quick

AVITA Medical, Valencia, CA, USA 
LOS compared to SOC across expected patient profiles and scenarios. In aggregate, ASCS treatment saves a burn center $14-17.3 \%$ annually. Results are sensitive to, but remain robust across, changing assumptions for relative impact of ASCS use on LOS, procedure time, and number of procedures.

Conclusions: Use of ASCS compared to SOC reduces hospital costs and LOS of severe burns in the USA.

Funding: AVITA Medical.

Keywords: Autologous cell harvesting device; Budget impact; Burn care; Cost-effectiveness; Dermatology; Skin graft; Split-thickness

\section{INTRODUCTION}

Burn injuries represent approximately $1 \%$ of non-fatal injuries among US civilians [1], with nearly 500,000 burn victims seeking medical care and approximately 40,000 patients requiring hospitalization [2]. As a result of complex and individualized treatment, the management of severe burns requires a high intensity of healthcare resource utilization and long inpatient stays that lead to high medical care costs. The economic burden of burns in the USA is significant, estimated at over $\$ 7.9$ billion per year (2018, inflated from 2010) for hospitalizations, emergency department visits, and deaths [3].

Burn management has evolved with the integration of new technologies and treatment paradigms into routine care, resulting in significant declines in the number of burn fatalities [4]. In past decades, mortality was common for burns greater than $20 \%$ total body surface area (TBSA) [4]. Today, new interventions have allowed patients with burns covering $90 \%$ of their bodies to survive [5]. However, there remain limited alternatives for effectively managing patients, minimizing morbidity, and mitigating the substantial cost of burn injury for severe burns requiring surgical intervention [6].

RECELL ${ }^{\circledR}$ Autologous Cell Harvesting Device (ACHD) (AVITA Medical, Valencia, CA, USA) is an innovative technology recently FDA- approved that allows the rapid preparation of autologous skin cell suspension (ASCS) at the point-of-care for treatment of acute thermal burn wounds $[7,8]$. ASCS may be applied either as a primary intervention for deep partialthickness (DPT) burns with confluent dermis, or as an adjunct to widely meshed split-thickness skin grafts (STSG) for mixed-depth and fullthickness (FT) burns (hereafter named FT/ mixed-depth). Published clinical trials, compassionate use data, and real-world evidence point to economic and clinical benefits of ASCS use in burn care. Recent clinical trials illustrate that use of ASCS significantly minimizes donor skin harvesting requirements, enhances re-epithelialization of widely meshed skin grafts, and may decrease the need for follow-up reconstructive procedures [8-10]. Furthermore, when compared to STSG, analysis of real-world burn center records demonstrates that use of ACHDgenerated ASCS, in isolation and in combination with STSG, can reduce hospital length of stay (LOS) for severe burn patients [7, 11].

In the current environment of healthcare resource scarcity, hospitals must increasingly consider both clinical efficacy as well as budgetimpact when deciding whether to adopt new products. However, the complexity of burn care presents a challenge when evaluating value for money for new interventions. Numerous aspects of practice patterns vary, including the timing of burn-depth diagnosis, excision technique and timing, use of temporary dressings, dermal substitutes, autografting technique, intensity of inpatient rehabilitation, and criteria for patient discharge and outpatient follow-up [12]. As a result, it is difficult to design and implement randomized controlled trials (RCTs) or other direct studies of interventions that are applicable across the spectrum of patient profiles, burn types, and provider practice patterns. In this complex clinical scenario, therefore, it can be useful to apply modeling methods to explore the possible range of expected health impact and economic outcomes.

To the authors' knowledge, there is no validated economic model of the inpatient burn care pathway available for assessment of health economic impact of new interventions versus current standard of care (SOC). Recognizing the 
importance of both the economic and clinical impacts of ASCS use in burn care, this study utilizes a health economic modeling approach to represent current inpatient management of burns and to capture the expected impact of ASCS compared to SOC. Specifically, we estimate the cost-effectiveness and burn center budget-impact for the use of ASCS compared to conventional STSG.

\section{METHODS}

\section{Structure}

A burn center perspective cost-effectiveness model (CEM) of the burn care pathway (Fig. 1), known as the Burn-MCM (medical counter measure) Effectiveness Assessment Cost Outcomes Nexus (BEACON) model, evaluates a single inpatient stay for the management of a severe burn.

The model takes patient characteristics as input and then utilizes linked, sequential decision trees across multiple modules to estimate the clinical and economic outcomes associated with each phase of care, and overall, during inpatient care. Each module represents a sequential progression through key clinical phases of burn care, including wound assessment, debridement/excision, temporary coverage, and definitive closure. An overview of the burn care pathway and core assumptions is detailed in supplementary materials. In brief, a cohort of patients enters the model at the time of wound assessment, and the depth of wound is determined. To simplify these analyses, it is assumed that all patients are diagnosed correctly in the wound assessment phase. Following wound assessment, a patient moves to debridement/excision where the wound is cleaned and may also be excised for removal of necrotic tissue. For subsequent phases of burn care, management options vary on the basis of burn depth. A patient diagnosed with DPT or FT/mixeddepth burn may receive temporary coverage during the waiting period before the next treatment takes place or for dermal regeneration. For this ASCS-focused analysis, we do not explicitly model temporary coverage interventions.

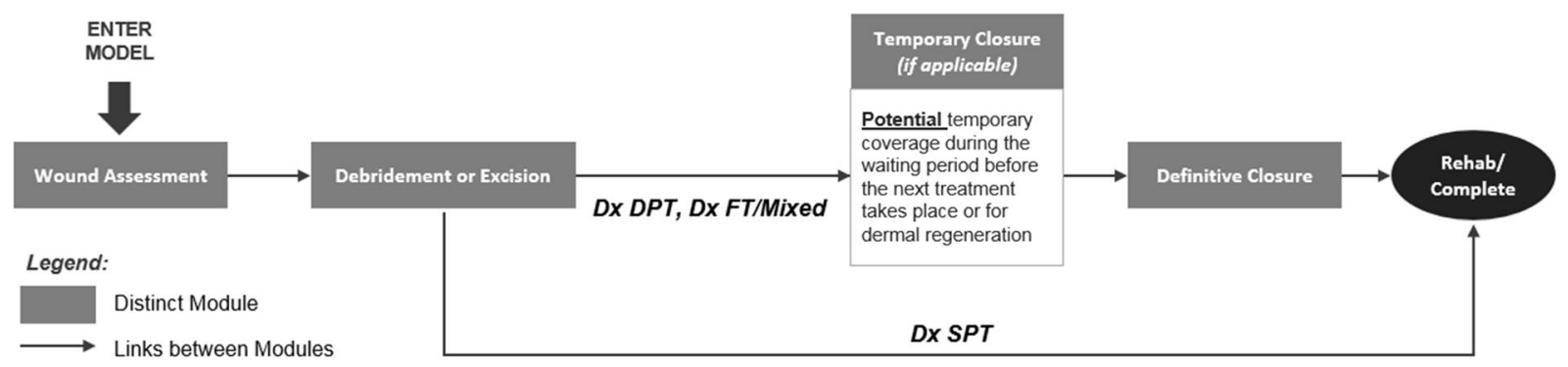

Acronyms: Dx - diagnose, DPT - deep partial-thickness, FT - full-thickness, SPT - superficial partial-thickness

Fig. 1 Burn model diagram. Wound assessment-the depth of wound is assessed, and a patient's wounds are diagnosed in terms of depth. Debridement or excision - per US standard practice, DPT and FT/mixed-depth burns are surgically excised in the operating room until viable bleeding tissue is reached to prepare the wound for definitive closure. SPT burns are assumed to be debrided to remove devitalized tissue and treated using conservative management without surgery. Temporary closure-for this ASCS-focused analysis, we implicitly capture the impact of temporary coverage on LOS and cost through predictive equations derived from burn center data. However, we do not explicitly model the individual unit costs or performance of potential temporary coverage (including dermal regeneration) interventions. Note that interventions for temporary coverage are not explicitly modeled at this time; however, their impact on total cost and length of stay is implicitly considered with the NBR predictive equations. Definitive closure - in this phase of burn care, wounds that are diagnosed as requiring surgery for definitive closure (DPT, FT/mixed-depth) and receive STSG or treatment with ASCS (with or without STSG). Rehabilitation-though not a discrete phase, the model evaluates resources to capture key inpatient rehabilitation cost as well as the proportion of patients requiring contracture operations 
For phases of burn care not impacted by ASCS treatment (namely, wound assessment, debridement/excision, temporary coverage), non-differential SOC was assumed to provide an evidence-based benchmark for patient outcomes under SOC in current clinical practice (as outcomes such as LOS and total cost are affected by all phases of care) and to isolate the incremental impact of ASCS use within the overall context of burn care management.

The budget impact model (BIM) builds on the CEM to capture the impact of interventions on costs and patient outcomes for a burn center overall, accounting for key drivers specific to the burn center, such as the expected patient mix by burn depth, TBSA burned, and other individual patient characteristics. The model compares costs for the two treatment pathways (with and without the use of ASCS) to isolate the likely shift in costs related to ASCS.

The BIM samples from normal distributions around patient and burn characteristics from the American Burn Association National Burn Repository (NBR) to generate 200 proxy patient profiles representing a real-world population of patients treated annually in the inpatient setting. For each unique patient profile, the detailed CEM estimates patient-level outcomes (e.g., LOS, cost, number of surgical operations). A Monte Carlo simulation is then used to generate the 200 profiles 100 times, enhancing the stability and precision of results. The combination of a Monte Carlo approach and sampled patient profiles enables the model to test the impact of an intervention, given variation in patient characteristics (e.g., input variability). The BIM aggregates results across the profiles to calculate the total fiscal impact to a burn center for two treatment pathways, considering the likely number of patients in each unique profile.

This article does not contain any new studies with human or animal subjects performed by any of the authors.

\section{Patient Profile}

The target population for the model is adults (average 42 years of age), with severe burns of TBSA $\geq 10 \%$ receiving inpatient care, where
DPT and FT/mixed-depth burns are eligible for ASCS.

Model inputs for additional factors that influence patient outcomes, such as inhalation injury, infection [hospital-acquired infection (HAI) and other infections], and sex, were derived from analysis of the NBR. The NBR includes 10 years of cumulative data from burn centers, representing the largest data resource for burn injuries in the USA including demographic, injury, and outcome information. Leveraging the NBR data (version 8.0, 2002-2011), analyses were performed on a sample of 21,175 surviving patients for whom key data points were available. All analyses leveraging the NBR were based on patients with TBSA between $10 \%$ and $60 \%$ to reduce a tail effect where outlier patients with high TBSA may skew predicted estimates. The NBR includes only relative burn size information (as $\%$ TBSA). Therefore, the National Health and Nutrition Survey (NHANES) [13] 2014-2016 was analyzed to determine the average absolute body surface areas (BSA, $\mathrm{cm}^{2}$ ) for male and female subjects in the USA to convert percentage TBSA from the NBR to an average size of burns in terms of square centimeters, needed for cost calculations in the model.

\section{Clinical Inputs}

Clinical inputs were derived from RCTs, a survey of eight burn surgeons, NBR database analyses, and in-depth interviews with four experienced burn surgeons. Further, all model assumptions were vetted by one or more burn surgeons, and the output of the model was benchmarked against real-world data to validate the modeling structure and ensure clinical validity [14] (Table 1).

To ensure the model accurately accounts for patient characteristics when predicting outcomes and costs, patient LOS associated with STSG treatment was estimated using the NBR. LOS was predicted via a regression controlling for TBSA, TBSA of partial-thickness burn, age (linear, squared and cubed to account for nonlinearity), HAI, other infection, inhalation injury, sex, whether or not they had one or 
Table 1 Key clinical inputs

Variable
Wound assessment
Time until wound depth diagnosed/first
procedure

procedure

\section{Debridement/excision}

Average number of non-excisional debridement procedures (FT/mixeddepth) Average number of non-excisional
debridement procedures (DPT)

Time per debridement procedure (mins)

Average number of excision procedures

(FT/mixed-depth)

Average number of excision procedures

(DPT)

Average time per excision procedure (mins)

\section{Definitive closure}

Conservative approximation: number of autograft operations (STSG, FT/mixeddepth \& DPT)

\section{Patient profile}

TBSA $10-20 \%$

TBSA $21 \%+$

TBSA $10 \%$

TBSA $20 \%$

TBSA $30 \%$

TBSA $40 \%$

TBSA $10 \%$

TBSA $20 \%$

TBSA $30 \%$

TBSA $40 \%$

TBSA $10 \%$

TBSA 20\%

TBSA 30\%

TBSA $40 \%$

TBSA $10 \%$

TBSA $20 \%$

TBSA $30 \%$

TBSA $40 \%$

TBSA $10 \%$

TBSA 20\%

TBSA 30\%

TBSA $40 \%$

TBSA $10 \%$

TBSA 20\%

TBSA 30\%

TBSA $40 \%$

TBSA 10-20\%

TBSA $21 \%+$
4.938 days

4.525 days

0.48

0.32

0.30

0.32

0.56

0.47

0.52

References

Burn surgeon survey (data on file)

NBR database analyses (data on file) 0.62 19 38 47 62

NBR database 2.98 3.57 analyses (data on file)

Burn centers 75 (data on file) (data on file) 
Table 1 continued

\begin{tabular}{|c|c|c|c|}
\hline Variable & Patient profile & Input & References \\
\hline \multirow{4}{*}{$\begin{array}{l}\text { NBR national average: number of autograft } \\
\text { operations (STSG, FT/mixed-depth) }\end{array}$} & TBSA $10 \%$ & 2.46 & \multirow{8}{*}{$\begin{array}{l}\text { NBR database } \\
\text { analyses (data } \\
\text { on file) }\end{array}$} \\
\hline & TBSA $20 \%$ & 3.14 & \\
\hline & TBSA $30 \%$ & 3.83 & \\
\hline & TBSA $40 \%$ & 4.54 & \\
\hline \multirow{4}{*}{$\begin{array}{l}\text { NBR national average: number of } \\
\text { autograft operations (STSG, DPT) }\end{array}$} & TBSA $10 \%$ & 2.23 & \\
\hline & TBSA $20 \%$ & 2.69 & \\
\hline & TBSA $30 \%$ & 3.15 & \\
\hline & TBSA $40 \%$ & 3.63 & \\
\hline \multirow{2}{*}{$\begin{array}{l}\text { Donor site size for STSG treatment (\% of } \\
\text { burn) }\end{array}$} & TBSA $10-39 \%$ & $61.1 \%$ & Gravante [9] \\
\hline & TBSA $40 \%+$ & $25 \%$ & Holmes [8] \\
\hline $\begin{array}{l}\text { Donor site size for ASCS treatment (\% of } \\
\text { burn) }\end{array}$ & All TBSA & $1.3 \%$ & Gravante [9] \\
\hline \multirow{2}{*}{$\begin{array}{l}\text { Donor site size ASCS + STSG (\% of } \\
\text { burn) }{ }^{*}\end{array}$} & TBSA $10-39 \%$ & $41.5 \%$ & Holmes [8] \\
\hline & TBSA $40 \%+$ & $17 \%$ & Holmes [8] \\
\hline \multirow[t]{2}{*}{ Autograft operative time (mins) } & Burn wound site & 1.6 per TBSA & \multirow{2}{*}{$\begin{array}{l}\text { Burn surgeon } \\
\text { survey (data } \\
\text { on file) }\end{array}$} \\
\hline & Donor site & 2.1 per TBSA & \\
\hline \multicolumn{4}{|l|}{ Other } \\
\hline \multirow{2}{*}{$\begin{array}{l}\text { Odds ratio for LOS for ASCS relative to } \\
\text { SOC (up to } 40 \% \text { TBSA) }\end{array}$} & $\mathrm{DPT}$ & 0.70 & Park [11] \\
\hline & FT/Mixed & 0.98 & Park [11] \\
\hline $\begin{array}{l}\text { Odds ratio for LOS for ASCS relative to } \\
\text { SOC (over } 40 \% \text { TBSA) }\end{array}$ & DPT \& FT/mixed & 0.53 & Holmes [19] \\
\hline \multirow{2}{*}{$\begin{array}{l}\text { Proportion of patients requiring } \\
\text { contracture procedures }(\%)\end{array}$} & STSG & $37.5 \%$ & \multirow[t]{2}{*}{ Gravante [9] } \\
\hline & ASCS & $28.6 \%$ & \\
\hline \multirow[t]{2}{*}{ Blood requirements per \% TBSA $(\mathrm{ml})$} & Excision & 20.51 & \multirow[t]{2}{*}{ Luo $[22]$} \\
\hline & STSG & 32.83 & \\
\hline
\end{tabular}

*Assumption based on meshing ratio of 4:1 for STSG and for ASCS + STSG, relative reduction in donor site size for ASCS + STSG from Holmes 2018 [8]

more grafting procedures, and diabetes status [15]. Similarly, the numbers of non-excisional debridements (ICD-9: 86.28) and excisional debridements (ICD-9: 86.22) are predicted using predictive equations derived from the NBR, based on patient characteristics.

\section{Definitive Closure}

The impact of the use of ASCS is modeled in the definitive closure module. In this phase of care, wounds diagnosed as requiring surgery for timely closure (DPT, FT/mixed-depth) can receive ASCS or SOC. For burns treated with 
ASCS, the model assumes ASCS alone for DPT burns and ASCS with meshed STSG for FT/ mixed-depth burns, while all SOC patients receive conventional STSG.

Other key differences in patient management for ASCS versus SOC include number of definitive closure procedures, procedure time, size of donor site, and LOS. In all scenarios considered in the model, definitive closure (healing) using ASCS is assumed to require one surgical procedure (i.e., healing is achieved with a single ASCS treatment for a given patient) [7-10]. As outlined below, model assumptions for the number of procedures for SOC were developed to capture the variability in practice patterns.

As highlighted during burn surgeon interviews and analysis of NBR data, surgical practices for definitive closure vary on the basis of surgeon preference, as well as patient and burn center characteristics. Therefore, estimated costeffectiveness of ASCS is presented for two scenarios to account for likely real-world variation in SOC STSG practices: (1) a conservative approximation, and (2) an NBR-based national average. The conservative approximation SOC scenario assumes a single conventional autografting procedure with STSG for patients with TBSA $\leq 20 \%$ and two conventional autografting procedures with STSG for patients with TBSA $>20 \%$. For the NBR national average scenario, the number of procedures to achieve definitive closure via conventional autografting with STSG (by burn depth and TBSA) was predicted using NBR data, with conventional autografting procedures identified via ICD-9 codes $86.61,86.62,86.63$, and 86.69 [16]. It was assumed that multiple codes were performed in the same operation, and therefore the maximum count of a single conventional autografting ICD-9 procedure code represented would estimate the number of SOC definitive closure procedures.

Information on donor site size harvested for ASCS and SOC STSG, as well as the proportion of patients requiring a contracture release procedure associated with ASCS treatment, is based on RCTs [8-10]. The number of ACHD devices used to prepare ASCS is determined by the percentage TBSA burned. On the basis of product information [17] one device can be used to prepare up to $24 \mathrm{ml}$ of ASCS, which treats up to $1920 \mathrm{~cm}^{2}$ of burn wound. For patients with TBSA up to $40 \%$, Gravante et al. demonstrated that average donor site per percent TBSA of burn was $61.1 \%$ for SOC STSG and $1.25 \%$ for ASCS [9]. For patients with TBSA over $40 \%$, size of donor site per percent TBSA was estimated at $25 \%$ based on a survey of eight practicing surgeons for SOC STSG, with a 32\% reduction (relative to SOC STSG) due to use of ASCS based on randomized clinical data [10]. Impact of reduced donor site in the model is captured via reduced time to harvest donor skin and reduced time and resources to dress the donor site. In addition to reducing size of the donor site per percent TBSA, use of ASCS is associated with reduced patient pain related to donor site harvesting and reduced number of additional procedures arising from limited availability of donor sites to support SOC STSG [10].

The impact of ASCS on LOS was derived from published, real-world evidence from Australia [11] as the device for preparation of ASCS has only recently become available outside of clinical trials in the USA [18]. Specifically, to estimate the effect of ASCS on LOS, odds ratios (OR) for DPT (0.7) and FT/mixed-depth (0.98) burn depths were applied to SOC-based LOS, as estimated by the NBR equation for burns up to $40 \%$ TBSA [11]. For burns with TBSA $\geq 40 \%$, compassionate use data from the USA was used to inform the impact of ASCS treatment on LOS (OR 0.53) [19]. Routine daily patient care outside of the operating room was assumed the same for ASCS and STSG. As such, changes in LOS costs are representative of reduced LOS only and conservatively do not assume any change in costs per day outside of key procedures. Daily care included daily dressing changes for both the burn wounds and donor sites, performed by one nurse and one technician with an estimated $1 \mathrm{~min}$ of staff time per square centimeter of burn, as estimated by a survey of eight burn surgeons. For operating room costs, key differences related to ASCS use include reduced number of procedures as well as reduced time for donor skin harvesting and donor skin wound dressings. 
After definitive closure, the model accounts for inpatient rehabilitation costs. On the basis of burn surgeon input, for all patients, one physical therapy and one occupational therapy appointment was assumed for each day of inpatient stay. The model also accounts for the proportion of patients requiring surgery for contracture release, with rates for ASCS and SOC based on published clinical data $[8,9]$. For patients requiring contracture release, we assumed a 3-day hospital stay for the procedure but do not assume rehabilitation costs, thereby presenting a conservatively low estimate of the cost of contracture.

\section{Costs and Resource Use}

Key cost elements include staffing (nurses, scrub technicians), costs per day (or bed costs), operating room time and related resources, woundrelated resources (e.g., wound dressings), and the price of the device(s) used to prepare ASCS. The model results presented herein conservatively assumed that burn surgeon and anesthesiologist time is billed separately and does not represent a cost to the burn center. As a result of limitations in obtaining nuanced data on anesthesia cost by each inpatient procedure, a non-differential lump sum anesthesia cost was conservatively applied to all patients undergoing surgery regardless of receiving SOC or ASCS. The cost of ASCS use (per $1920 \mathrm{~cm}^{2}$ of burn wound) is based on the $\$ 7500$ list price of the ACHD used to prepare ASCS. Hospital resource use (e.g., materials, procedure time) and unit costs were derived from survey data obtained from three US burn centers. All unit costs represent 2017 USD and are reflective of average costs reported by surgery centers (Table 2 ).

\section{Analyses}

\section{Cost-Effectiveness Analysis}

The BEACON model is fully customizable to support use of individual burn center data. The results presented are based on national aggregate trends. The model predicts outcomes for patients with any TBSA; however, results presented herein focus on TBSA ranges that are
Table 2 Key cost and resource use inputs

\begin{tabular}{|c|c|c|}
\hline $\begin{array}{l}\text { Provider resource } \\
\text { use element }\end{array}$ & Unit & Cost (USD 2017) \\
\hline $\begin{array}{l}\text { Cost per day for burn } \\
\text { patients }\end{array}$ & Per day & $\$ 6795.00$ \\
\hline $\begin{array}{l}\text { Burn surgery } \\
\text { operating room } \\
\text { time }\end{array}$ & Per hour & $\$ 3720.00$ \\
\hline Nurse time & Per hour & $\$ 56.10$ \\
\hline Scrub tech time & Per hour & $\$ 39.00$ \\
\hline $\begin{array}{l}\text { Blood transfusion } \\
\text { (packed cells, whole } \\
\text { blood) }\end{array}$ & Per liter & $\$ 117.00$ \\
\hline Escharotomy & Per excision & $\$ 500.00$ \\
\hline $\begin{array}{l}\text { Wound dressings } \\
\text { inpatient }\end{array}$ & Per $\mathrm{cm}^{2}$ & $\$ 0.09$ \\
\hline Physical therapy & Per session & $\$ 21.75$ \\
\hline Occupational therapy & Per session & $\$ 15.75$ \\
\hline $\begin{array}{l}\text { Contracture surgery } \\
\text { first }\end{array}$ & $100 \mathrm{~cm}^{2}$ & $\$ 100.00$ \\
\hline $\begin{array}{l}\text { Contracture surgery } \\
\text { subsequent }\end{array}$ & $100 \mathrm{~cm}^{2}$ & $\$ 50.00$ \\
\hline Anesthesiology & Per patient & $\$ 2694.00$ \\
\hline $\begin{array}{l}\text { List price for } \\
\text { Autologous Cell } \\
\text { Harvesting Device } \\
\text { for preparation of } \\
\text { ASCS }\end{array}$ & Per device & $\$ 7500.00$ \\
\hline
\end{tabular}

common in real-world care [20]. Specifically, results are reported for adult patients (average age 42 years) with TBSA 10\%, 20\%, 30\%, and $40 \%$ for DPT and FT/mixed-depth, controlling for comorbidities as derived from NBR data. The selected patient profiles for TBSA and burn depths were chosen to illustrate a range of costeffectiveness outcomes with ASCS use.

While overall patient characteristics were held consistent across model runs for DPT and FT/mixed-depth burn depths, underlying model inputs were varied (per Table 1) to account for 
the impact of wound depth on LOS and amount of donor skin harvested (and associated impact on surgery time). Two scenarios were analyzed-assuming a conservative approximation and using NBR-based national averages to predict number of SOC STSG procedures. One-way sensitivity analysis (OWSA) was conducted for each patient profile (Table 3).

\section{Budget Impact Analysis}

To estimate budget impact, a burn center treating 200 patients annually was simulated. Consistent with the CEM, individual patient characteristics were extracted from the NBR, including average age, sex, and comorbidities. This information represents national aggregate data on the mix of patient types, burn depth, and TBSA for a burn center. As highlighted in Table 3, the BIM includes the full range of patient and burn characteristics expected to present in the USA. Therefore, while the target CEM profiles described above highlight the range in outcomes across potential patient and burn types, the BIM considers the relative mix of TBSA ranges.

The BIM requires categorization of patients into discrete burn depths of superficial partialthickness (SPT), DPT, and FT/mixed-depth. Burn depth is not a discrete variable in the NBR; however, continuous variables of TBSA of FT and PT are reported. These variables were used to categorize a patient as FT/mixed-depth if

Table 3 Cost-effectiveness and budget impact patient profiles Source: Inputs based on analysis of NBR data

\begin{tabular}{lllll}
\hline \multicolumn{4}{l}{ Details of patient profiles for the cost-effectiveness model } \\
\hline \multicolumn{4}{c}{ TBSA } \\
\hline Patient characteristics & $10 \%$ & $20 \%$ & $30 \%$ & $40 \%$ \\
Female (\%) & $26 \%$ & $23 \%$ & $27 \%$ & $27 \%$ \\
BSA $\left(\mathrm{cm}^{2}\right)$ & 19,808 & 19,856 & 19,788 & 19,783 \\
Size of burn $\left(\mathrm{cm}^{2}\right)$ & 1981 & 3971 & 5936 & 7913 \\
Comorbidities & & & & \\
Inhalation injury (\%) & $4 \%$ & $9 \%$ & $13 \%$ & $25 \%$ \\
Hospital-acquired infection (HAI) (\%) & $1 \%$ & $3 \%$ & $4 \%$ & $9 \%$ \\
Other infection (\%) & $2 \%$ & $5 \%$ & $4 \%$ & $5 \%$ \\
Diabetes (\%) & $6 \%$ & $6 \%$ & $3 \%$ & $4 \%$ \\
\hline
\end{tabular}

Details of default settings for a burn center with 200 adult patient annually

\begin{tabular}{llll}
\hline & $\begin{array}{l}\text { Full-thickness/mixed-depth } \\
\text { No. Patients (\%) }\end{array}$ & $\begin{array}{l}\text { Deep partial-thickness } \\
\text { No. Patients (\%) }\end{array}$ & $\begin{array}{l}\text { Superficial partial-thickness } \\
\text { No. Patients (\%) }\end{array}$ \\
\hline $\begin{array}{l}\text { Wound depth distribution } \\
\text { Proportion of burns }\end{array}$ & $40(20 \%)$ & $58(29 \%)$ & $102(51 \%)$ \\
TBSA 40\%+ (average 48\%) & $5(13 \%)$ & $5(8 \%)$ & $7(7 \%)$ \\
TBSA 21-40\% (average 28\%) & $15(38 \%)$ & $20(35 \%)$ & $26(25 \%)$ \\
TBSA 10-20\% (average 15\%) & $19(49 \%)$ & $33(57 \%)$ & $69(68 \%)$
\end{tabular}

a Superficial partial-thickness patients receive no STSG or ASCS, as they are assumed to heal within 21 days. Note: May not sum to $100 \%$ because of rounding of number of patients in simulation 
$50 \%+$ of their burn was FT depth and as SPT if they had zero surgical procedures in the NBR, with remaining burns classified as DPT.

\section{RESULTS}

\section{Cost-Effectiveness Analysis}

In the conservative approximation scenario of ASCS use, spend was cost-neutral $(<2 \%)$ or costsaving in all profiles. Using the NBR national averages to predict number of STSG operations in an alternate scenario, all patient profiles showed cost savings with use of ASCS. In both scenarios, reduced number of definitive closure procedures enabled through use of ASCS use was driven by reduced need for donor skin. Accordingly, mitigating availability of donor skin as a limiting factor, fewer surgical operations were required to achieve definitive closure. A key finding was that cost savings increase as burn size increases owing to overall reduction in the number of operations, dressing time, and associated costs (Table 4).

LOS was reduced for all patient profiles modeled, but the relative shift in LOS was most favorable for large burns. The OR for LOS with ASCS, relative to NBR-derived SOC estimates, was most favorable for DPT burns as well as for burns with TBSA of $40 \%$ or more, which led to greater reductions in LOS and associated inpatient costs for these patients. Notably, for FT/ mixed-depth burns of $40 \%$ TBSA, the projected reduction in LOS was almost 28 days (SOC, 59 days; ASCS, 31 days). Further, large relative LOS reductions were seen across all TBSA ranges for DPT, with ASCS-reductions in LOS increasing along with increases in TBSA percentages. Across all patient profiles, the use of ASCS translates to roughly a $20 \%$ reduction in rehabilitation costs $(\sim \$ 2000$ savings per acute care stay), due to a reduced proportion of patients requiring surgical procedures for contracture release and reduced number of days as inpatients with physical therapy and occupational therapy visits.

In OWSA, the OR for ASCS impact on LOS for each strategy is the primary driver of results for all depths and burn sizes. Additional influential variables include the size of the donor site and the number of operations for SOC. Nevertheless, for all patient profiles, the use of ASCS consistently led to cost savings or cost-neutral results when varying model inputs across expected high and low ranges, which suggests that model results remain robust across expected uncertainties or variations in individual model parameters. OWSA diagrams can be found in supplementary materials.

\section{Budget Impact Analysis}

Aggregating patient profiles to view results for a burn center, use of ASCS is expected to reduce overall costs by an estimated $14-17.3 \%$ annually. Under the conservative scenario for estimating SOC STSG procedures, use of ASCS reduced costs by an estimated 14\% (\$5.3 million for the burn center, average $\$ 26.6$ thousand per patient). When estimating SOC STSG procedures per NBR averages, overall relative savings increased to $17.3 \%$ ( $\$ 6.8$ million for the burn center, $\$ 34.1$ thousand per patient). Reductions in costs are driven by reduced number and duration of procedures performed for definitive closure, change in LOS, and reduced rehabilitation needs (Table 5). Notably, use of ASCS led to an estimated $32 \%$ and $37 \%$ reduction in definitive closure procedures relative to SOC for the conservative and NBR scenarios, respectively.

\section{DISCUSSION}

This study evaluated the impact of ASCS on patient LOS, number and duration of definitive closure procedures, inpatient resource use, and the estimated cost impact to a burn center for treatment of severe burns in the USA. At a patient level, use of ASCS for burn treatment (regardless of burn depth) was predicted to be cost saving or cost neutral vs SOC when applying the conservative approximation (for SOC procedures) and consistently cost saving for all patient profiles when applying the NBR predictive equations (for SOC procedures). All sensitivity analyses continued to show cost-saving or at least cost-neutral results, demonstrating that 


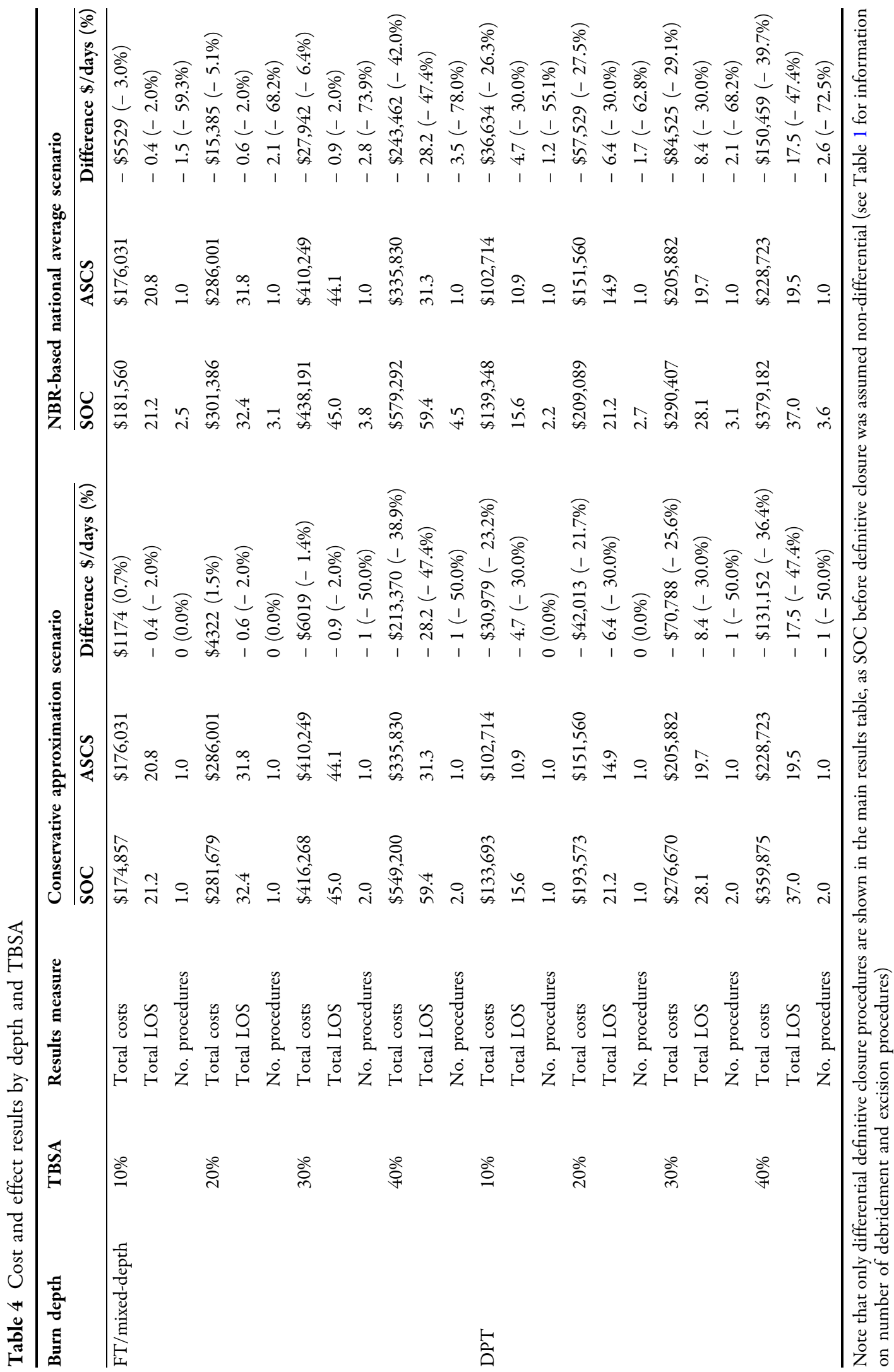




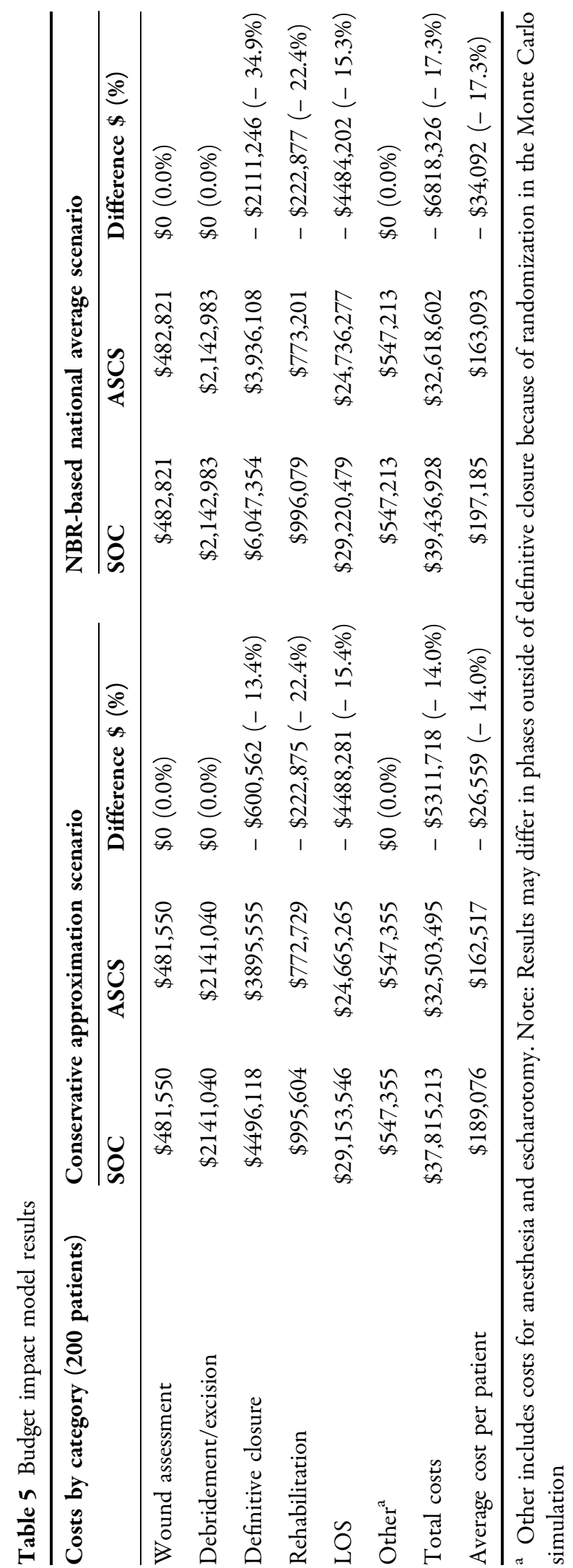

the core model results remain robust across expected uncertainties in individual model parameters.

Results of the CEM illustrate consistent costsaving results across a range of individual patient profiles, as highlighted by the burn depth and TBSA ranges presented. Leveraging the individual patient results from the CEM, the BIM considered the mix of patients and burn characteristics expected to present in the USA annually, concluding projected net savings to a burn center overall.

The underlying clinical driver associated with ASCS use is the reduced requirement for harvesting of donor skin, which leads to a reduced number of procedures and faster healing time [8-10]. While favorable results were observed across a range of patient profiles, the modeled impact of ASCS on LOS is likely conservative. Using the Australian real-world data, use of ASCS alone for DPT burns allowed for a greater reduction in LOS (OR 0.7) compared to ASCS with meshed-STSG for FT/mixed depth burns (OR 0.98) [11]. However, the higher relative OR for FT/mixed depth burns (0.98) may be conservatively biased given the majority of patients (92\%) had TBSA less than 20\%. The number of procedures needed for definitive closure for SOC increases with TBSA and, therefore, the benefits of a single procedure for ASCS also increased at the same time as expected LOS reductions. This trend of increased ASCS benefits with higher TBSA for FT/mixed depth wounds was also supported by US-based compassionate use data. Specifically, for patients with an average TBSA of $62 \%$ (range 40-91\%) use of ASCS with meshed-STSG showed an OR of 0.53 compared to SOC [19]. Therefore, it is expected that the impact of ASCS on LOS for FT/mixed depth burns may be greater than estimated in this analysis.

As with any modeling exercise, this study is subject to limitations relevant to interpretation of results. The primary limitation is that data comparing LOS for ASCS treatment versus SOC, outside of compassionate use and within-in patient controls, was not available in the USA. Therefore, we estimated the LOS for SOC patients in the US setting, and then applied the OR derived from a real-world study from 
Australia to estimate the impact of ASCS versus SOC for burns less than $40 \%$ TBSA. While the use of a relative effect in the form of an odds ratio follows best practices for modeling, it should be noted that we do not explicitly address any underlying differences between the US and Australian health systems that may impact the ASCS-related shift in LOS. For patients with $40 \%+$ TBSA, data from a single burn center were used that found a $47 \%$ reduction in LOS for patients receiving ASCS treatment compared to age and burn severity matched controls in a limited compassionate use sample ( $\sim 10$ adult patients) [19]. Nevertheless, results and assumptions have been verified by US burn surgeons. Furthermore, substantial and consistent clinical and financial benefits from use of ASCS in compassionate use experience in the USA has been reported [19].

Secondly, information about the costs and timing of procedures were obtained from a small sample of burn centers or from a survey of burn surgeons. These represent average costs as reported by the centers and, therefore, do not explicitly address the likely range in true paid costs when considering the mix of insurance types across patients. As mentioned earlier, surgical management of burns varies across burn centers and surgeons. Accordingly, staff and healthcare costs, as well as definitive closure procedure times, may also vary. Conclusions from the OWSA suggest that results remain robust across expected, known variations as well as potential shifts in costs due to insurance status of patients. Furthermore, the use of random sampling from distributions in the BIM highlights that conclusions also remain robust across variations in key inputs. The authors also conducted an external benchmark against predicted costs across patient profiles in the NBR to check validity of final results, concluding that predicted costs for SOC were consistent with NBR data [15].

Finally, several simplifying assumptions were made to develop a transparent, flexible model. First, individual unit costs and temporary coverage interventions (e.g., allograft, xenograft, skin substitute, or dermal analogs) were not explicitly considered. Temporary closure costs and patient impacts are only implicitly captured via the NBR-based predictive equations. Next, as is the case with clinical practice, the model assumes correct diagnosis when determining pathways for a diagnosed burn. Accuracy of burn depth diagnosis varies on the basis of burn center practices as well as timing of diagnosis, and published literature suggests that inaccurate diagnosis can occur, especially for SPT and DPT burns [21]. The most important impact of this assumption on model results is that the number of DPT burns eligible for ASCS (either misdiagnosed SPT or true DPT burns) may be uncertain. However, given that the use of ASCS is expected to result in savings for DPT burns, some amount of inherent misdiagnosis likely leads to an underestimate of cost savings for any incorrectly diagnosed SPT burns. Further, the NBR does not code diagnosis changes (i.e., burn depth conversion) over time and, therefore, does not highlight whether diagnoses were correct or incorrect. However, the predictive equations derived from the NBR implicitly capture the effects of how incorrectly diagnosed patients impact average outcomes for procedures and LOS. Also, the model assumes only one procedure for a patient with ASCS. While this is consistent with trends seen in real-world use in Australia as well as early clinical trial data [7], there may be instances when patients undergo more than one procedure given very high TBSA or provider preferences. Finally, the model does explicitly consider the cost of retreatment for ASCS or SOC, but the impact of retreatment on LOS may be implicitly captured in the NBR predictive equations for LOS.

Although the aforementioned limitations exist, best-practice modeling methods were used, and key assumptions were validated by burn surgeons, thereby ensuring that the analytic conclusions are clinically valid and useful to the burn community.

\section{CONCLUSIONS}

The BEACON model was developed to facilitate evaluation of cost-effectiveness of new interventions within burn care, as measured by the clinical outcomes and relative costs for management of burns in the USA. As a first step, the 
model was used to estimate the potential impact of treatment with ASCS for inpatient burn management for individual patient profiles and for burn centers, given current SOC practice patterns and the distribution of patient characteristics seen nationally. Overall, ASCS use reduces costs associated with the current treatment of severe burns, with greater savings seen in larger FT/mixed-depth burns and across all DPT burns. The cost savings are due to reductions in LOS, the number of operations required to close the burn wound, the donor site size, and associated donor site wound care.

Future analyses should seek to replace LOS parameters with real-world data for the USA across a broader range of patient profiles, and to obtain more data on costs and the timing of procedures from more hospitals to improve generalizability. Furthermore, information from individual burn centers could be integrated to identify how ASCS treatment is likely to impact costs and resource use given their current SOC practices. Finally, given that the model captures the full spectrum of burn care, future analyses could leverage the modeling framework to evaluate additional new interventions, alone or in combination with ASCS treatment, and thereby estimating the synergistic impacts of different interventions on the cost of burn care.

\section{ACKNOWLEDGEMENTS}

Funding/Support. Sponsorship for this study and article processing charges were funded by Biomedical Advanced Research Development Authority (Contract HHSO100201500028C) and AVITA Medical, Valencia, CA, USA. All authors had full access to all of the data in this study and take complete responsibility for the integrity of the data and accuracy of the data analysis.

Authorship. All named authors meet the International Committee of Medical Journal Editors (ICMJE) criteria for authorship for this article, take responsibility for the integrity of the work as a whole, and have given their approval for this version to be published.
Disclosures. Stacey Kowal is an employee of IQVIA and received funding to conduct the research. Eliza Kruger was an employee of IQVIA and received funding to conduct the research. Pinar Bilir is an employee of IQVIA and received funding to conduct the research. Jeremiah Sparks is an employee of Avita Medical. Andrew Quick is an employee of Avita Medical. Katie Bush is an employee of Avita Medical. James Holmes received consultation fees for contributions to input collection and model validation. William Hickerson received consultation fees for contributions to input collection and model validation. Kevin Foster received consultation fees for contributions to input collection and model validation. Narayan Iyer is an employee of BARDA, the Office of Assistant Secretary for Preparedness and Response and the United States Department of Health and Human Services throughout the duration of the study. Scott Nystrom was an employee of Office of Assistant Secretary for Preparedness and Response, US Dept. of Health and Human Services. The publication of study results was not contingent on the sponsor's approval or censorship of the manuscript.

Compliance with Ethics Guidelines. This article does not contain any new studies with human or animal subjects performed by any of the authors.

Disclaimer for HHS. The findings and conclusions in this report are those of the author(s) and do not necessarily represent the views of the Department of Health and Human Services or its components.

Data availability. The datasets generated during and/or analyzed during the current study are available from the corresponding author on reasonable request.

Open Access. This article is distributed under the terms of the Creative Commons Attribution-NonCommercial 4.0 International License (http://creativecommons.org/licenses/ by-nc/4.0/), which permits any noncommercial use, distribution, and reproduction in any medium, provided you give appropriate credit 
to the original author(s) and the source, provide a link to the Creative Commons license, and indicate if changes were made.

\section{REFERENCES}

1. Pruitt B, Mason A. Epidemiological, demographic, and outcome characteristics of burn injury. In: Herndon D, editor. Total burn care. London: Saunders; 1996. p. 13.

2. American Burn Association. Burn incidence and treatment in the United States: 2016. 2016. http:// www.ameriburn.org/resources_factsheet.php. Accessed 3 Dec 2018.

3. CDC. 20 Leading causes of nonfatal injury, United States. 2015, All races, both sexes, disposition: all cases. 2015. https://webappa.cdc.gov/sasweb/ncipc/ nfilead.html. Accessed 5 Mar 2018.

4. Klein MB, Goverman J, Hayden DL, et al. Benchmarking outcomes in the critically injured burn patient. Ann Surg. 2014;259:833.

5. National Institutes of Health. Research portfolio online reporting tools. Burns and traumatic injury 2010. 2010. https://report.nih.gov/nihfactsheets/ Pdfs/BurnsandTraumaticInjury(NIGMS).pdf. Accessed 6 Apr 2017.

6. Hranjec T, Turrentine FE, Stukenborg G, et al. Burncenter quality improvement: are burn outcomes dependent on admitting facilities and is there a volume-outcome "sweet-spot"? Am Surg. 2012;78:559-66.

7. Lim J, Liew S, Chan H, et al. Is the length of time in acute burn surgery associated with poorer outcomes? Burns. 2014;40:235-40.

8. Holmes J, Molnar J, Carter J, et al. A comparative study of the ReCell ${ }^{\circledR}$ device and autologous spitthickness meshed skin graft in the treatment of acute burn injuries. J Burn Care Res. 2018;39:694-702.

9. Gravante G, Di Fede M, Araco A, et al. A randomized trial comparing ReCell $^{\circledR}$ system of epidermal cells delivery versus classic skin grafts for the treatment of deep partial thickness burns. Burns. 2007;33:966-72.

10. Holmes J, Molnar J, Shupp J, et al. Demonstration of the safety and effectiveness of the RECELL ${ }^{\circledR}$ system combined with split-thickness meshed autografts for the reduction of donor skin required to treat mixed-depth burn injuries. Burns. 2018. https://doi.org/10.1016/j.burns.2018.11.002.
11. Park JH, Heggie KM, Edgar DW, et al. Does the type of skin replacement surgery influence the rate of infection in acute burn injured patients? Burns. 2013;39:1386-90.

12. Israel JS, Greenhalgh DG, Gibson AL. Variations in burn excision and grafting: a survey of the American Burn Association. J Burn Care Res. 2017;38:e125-32.

13. CDC. National Health and Nutrition Survey (NHANES) 2013-14. 2014. https://wwwn.cdc.gov/ nchs/nhanes/continuousnhanes/default.aspx?Begin Year=2013. Accessed 17 June 2017.

14. Bilir P, Kruger E, Kowal S, et al. Abstract: Inpatient cost of acute care for severe burn patients: validation of economic model for adults and children. In: ISPOR 2018. Baltimore, 2018.

15. Kruger E, Bilir P, Kowal S, et al. Abstract: Relationship between patient characteristics and length of stay for severe burn patients: analysis of the American Burn Association National Burn Repository. In: ISPOR 2018. Baltimore, 2018.

16. American Burn Association. National Burn Repository Database Version 8.0. 2012.

17. Avita Medical. INSTRUCTIONS FOR USE: RECELL ${ }^{\circledR}$ Autologous cell harvesting device. 2018. https:// www.fda.gov/downloads/BiologicsBloodVaccines/ BloodBloodProducts/ApprovedProducts/Premarket ApprovalsPMAs/UCM621327.pdf. Accessed 7 Feb 2019.

18. FDA. September 20, 2018 Approval Order-RECELL Autologous cell harvesting device. 2018. https:// www.fda.gov/BiologicsBloodVaccines/BloodBlood Products/ApprovedProducts/PremarketApprovals PMAs/ucm621291.htm. Accessed 2 Feb 2018.

19. Holmes JH, Molnar JA, Williams JW, et al. Compassionate use of ReCell in large burns: a singlecenter U.S. experience. Auckland: ANZBA; 2016.

20. American Burn Association. National Burn Repository 2017 update: report of the data from 2008-2017. 2018. http://www.ameriburn.org/ resources_factsheet.php. Accessed 8 Jan 2019.

21. Hoeksema H, Van de Sijpe K, Tondu T, et al. Accuracy of early burn depth assessment by laser Doppler imaging on different days post burn. Burns. 2009;35:36-45.

22. Luo $G$, Fan $H$, Sun $W$, et al. Blood loss during extensive escharectomy and auto-microskin grafting in adult male major burn patients. Burns. 2011;37:790-3. 